\title{
Single-Stage Ti:sapphire-Pumped Deep-Infrared Femtosecond Optical Parametric Oscillator based on $\mathrm{CdSiP}_{2}$
}

\author{
Callum F. O’Donnell, ${ }^{1,2}$ S. Chaitanya Kumar, ${ }^{1}$ P. G. Schunemann, ${ }^{3}$ K. T. Zawilski, ${ }^{3}$ \\ and M. Ebrahim-Zadeh ${ }^{1,2,4}$ \\ ${ }^{I}$ Radiantis, Polígon Camí Ral, 08850 Gavà, Barcelona, Spain \\ ${ }^{2}$ ICFO-Institut de Ciencies Fotoniques, The Barcelona Institute of Science and Technology, 08860 Castelldefels, Barcelona, Spain \\ ${ }^{3}$ BAE Systems, Incorporated, MER15-1813, P.O. Box 868, Nashua, New Hampshire 03061-0868, USA \\ ${ }^{4}$ Institucio Catalana de Recerca i Estudis Avancats (ICREA), Passeig Lluis Companys 23, Barcelona 08010, Spain \\ Authore-mail address: majid.ebrahim@icfo.eu
}

\begin{abstract}
We report the first deep-infrared femtosecond $\mathrm{OPO}$ based on $\mathrm{CdSiP}_{2}$ synchronouslypumped directly by a KLM Ti:sapphire laser, tunable across $7508-8210 \mathrm{~nm}$, and generating as much as $12 \mathrm{~mW}$ at $7508 \mathrm{~nm}$ in good beam-quality.
\end{abstract}

OCIS codes: (190.4360) Nonlinear optics, devices; (190.7110) Ultrafast nonlinear optics; (190.4400) Nonlinear optics, materials; (190.4970) Parametric oscillators and amplifiers.

\section{Introduction}

The deep-infrared (deep-IR) window of the optical spectrum in 6-8 $\mu \mathrm{m}$ wavelength range in the molecular finger print region has a wealth of information pertaining to many molecules of importance involved in a variety of applications, from surgery [1] to explosives detection [2]. In the absence of conventional lasers at these wavelengths, access to this spectral region almost entirely relies on nonlinear frequency conversion sources. In particular, optical parametric oscillators (OPOs) are powerful and viable solid-state sources of coherent radiation, providing access to deep-IR spectral region with wide tunability. Such deep-IR OPOs are based on a specific class of infrared nonlinear materials such as $\mathrm{ZnGeP}_{2}$ (ZGP) and orientation-patterned GaAs (OP-GaAs). While these materials have a wide transparency extending deep in to the infrared, they require pumping beyond $2 \mu \mathrm{m}$ to avoid two-photon absorption. In order to overcome this limitation, recent efforts have been directed towards the development of novel nonlinear materials which can be driven by the well-established pump laser technology near $1 \mu \mathrm{m}$. The quest for such a nonlinear material recently led to the development of cadmium silicon phosphide, $\mathrm{CdSiP}_{2}$ (CSP) [3]. The large band gap, high optical quality, good thermal and optical properties with high effective nonlinear coefficient $\left(d_{\text {eff }} \sim 84.5\right.$ $\mathrm{pm} / \mathrm{V})$, and noncritical phase-matching $(\mathrm{NCPM})$, make it an attractive nonlinear material candidate for the generation of deep-IR wavelengths in the 6-8 $\mu \mathrm{m}$ range [4]. To date, a number of ultrafast pump laser technologies based on Yb-fiber [5], Yb:KGW [6], cascaded pumping internal to another OPO [7], as well as external tandem pumping using commercially available OPOs [8], have been deployed in combination with CSP as the nonlinear material to access the deep-IR spectral region. Here, we take another forward step in the development of deepinfrared femtosecond OPOs based on CSP by extending the usable pump laser technology to the Kerr-lens-modelocked (KLM) Ti:sapphire laser. In doing so, we demonstrate the shortest pump wavelength and shortest pulse duration so far used to drive CSP, the longest deep-IR idler wavelength generated at practical powers, and the first demonstration of angle tuning in CSP.

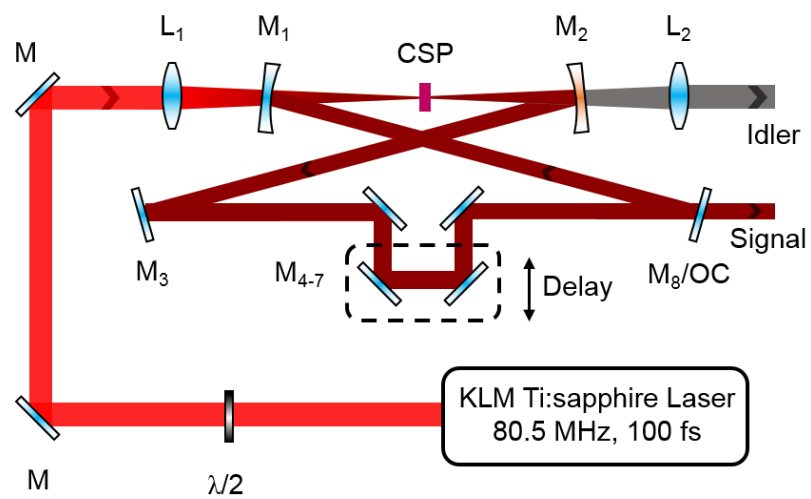

Fig. 1. Schematic of the experimental setup for the deep-IR CSP femtosecond OPO directly pumped by a KLM Ti:sapphire laser. 
The configuration of the CSP OPO is depicted in Fig. 1. The KLM Ti:sapphire pump source (Spectra-Physics, Mai Tai HP) produces transform limited pulses of $\sim 100 \mathrm{fs}$ at a wavelength of $1001 \mathrm{~nm}$, delivering an average power of $\sim 780 \mathrm{~mW}$ at a repetition rate of $80.5 \mathrm{MHz}$. The input polarization is adjusted by a half-wave plate $(\lambda / 2)$ for type 1 $\left(e \rightarrow o o\right.$ ) phase-matching in the CSP crystal, which is 1 - $\mathrm{mm}$-long with a $4 \mathrm{~mm} \times 5 \mathrm{~mm}$ aperture, and cut at $\theta=90^{\circ}$, $\left(\phi=45^{\circ}\right)$ for NCPM at normal incidence. The crystal end faces are both antireflection (AR)-coated, providing high transmission for the pump ( $T>99 \%$ at 1000-1080 nm), signal ( $T>99 \%$ over 1160-1240 nm), and idler ( $T>93 \%$ over $6000-7500 \mathrm{~nm})$. The ring cavity is comprised of two plano-concave mirrors $\left(\mathrm{M}_{1-2}\right)$ with radius of curvature, $r=100$ $\mathrm{mm}$, and six plane mirrors $\left(\mathrm{M}_{3-8}\right) . \mathrm{M}_{1}$ transmits the pump $(T \sim 80 \%$ at $1001 \mathrm{~nm})$ and is highly reflecting $(\mathrm{HR})$ for the signal $(R>99 \%$ across $1100-1300 \mathrm{~nm})$, and $\mathrm{M}_{2}$ is a 3-mm ZnSe substrate AR-coated for the deep-IR idler (T> 94\% across $6500-9000 \mathrm{~nm})$ and HR for the pump and signal ( $R>97 \%$ across $900-1500 \mathrm{~nm}) . \mathrm{M}_{8}$ functions as a $5 \%$ signal output coupler $(R=95 \%$ across $1100-1500 \mathrm{~nm})$. All remaining mirrors are fused silica substrate and HR for the signal $(R>99.8 \%$ across $980-1600 \mathrm{~nm})$ and optimized for minimal group-delay dispersion. The undepleted pump exits the cavity through $\mathrm{M}_{1}$, ensuring singly-resonant operation. $\mathrm{M}_{4-7}$ are arranged in a delay line, enabling cavity delay tuning of the output wavelengths without beam misalignment. The idler is collimated using a $\mathrm{CaF}_{2}$ lens $\mathrm{L}_{2}$ with $T$ > $70 \%$ over $6000-8500 \mathrm{~nm}$.

Figure 2(a) illustrates the potential deep-IR angle-tuning tuning range of CSP under critical phase-matching using established pump technology near $1 \mu \mathrm{m}$. As can be clearly seen, a wide angle-tuning range is available in the deepIR when pumping in the $\sim 1-1.064 \mu \mathrm{m}$ range. In Fig. 2(b), the measured spectrum of the KLM Ti:sapphire pump laser is compared to the calculated phase-matching acceptance in the 1-mm-long CSP crystal, confirming that the pump spectrum closely matches the parametric gain bandwidth, hence ensuring efficient interaction.
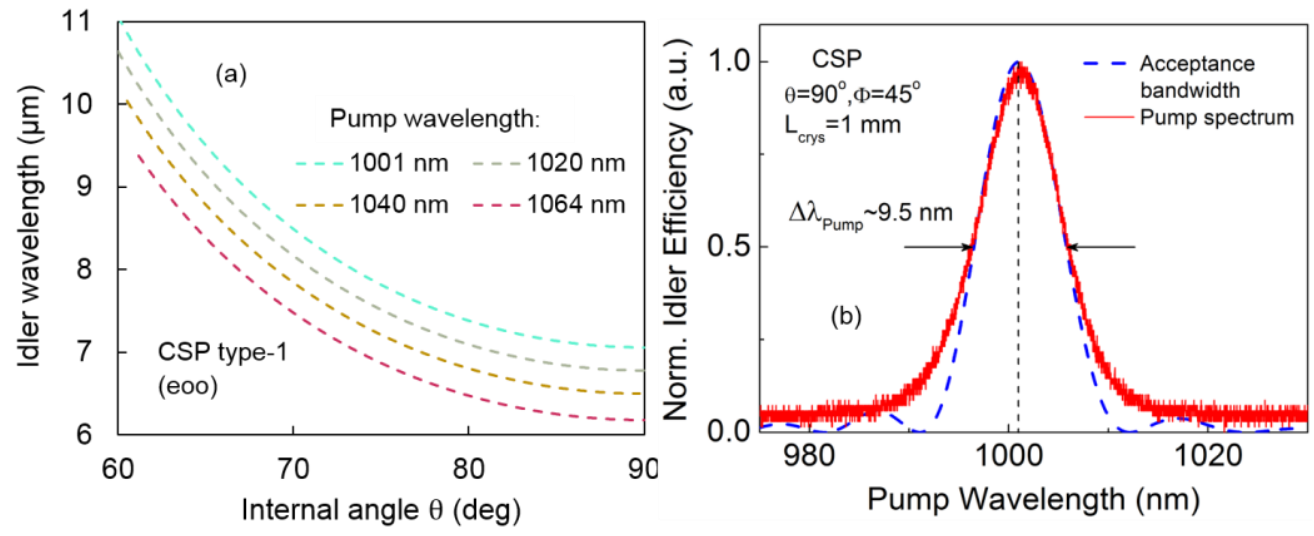

Fig. 2 (a) Comparison of available idler tuning using angle-tuned critical phase-matching, calculated using the relevant Sellmeier equations [3], for a Ti:sapphire pump at $1001 \mathrm{~nm}$ and previously demonstrated sources pumped at $\sim 1020 \mathrm{~nm}, 1040 \mathrm{~nm}$ and $1064 \mathrm{~nm}$. (b) Calculated pump acceptance bandwidth compared to measured signal spectrum.

In Fig. 3 we present the angle tuning range in the CSP femtosecond OPO for the pump wavelength at $1001 \mathrm{~nm}$, with the experimental wavelength data corresponding to minimum cavity delay. As can be clearly seen, the OPO is tunable across $1140-1155 \mathrm{~nm}$ in the near-IR signal, corresponding to a deep-IR idler tunability across $7508-8210 \mathrm{~nm}$ (over $702 \mathrm{~nm}$ ) by angular interrogation of the CSP crystal over 25 degrees. The solid curves represent the calculated angle tuning range using the relevant Sellmeier equations for the material [3]. The discrepancy between the experimental data and calculated tuning range can be attributed to strong self-phase-modulation in the cavity. As shown in the inset, the signal spectrum contains an extended tail towards longer wavelengths and the recorded spectral peak may lie away from the minimum of the phase-matching condition. With the implementation of intracavity dispersion compensation, we expect to achieve smooth spectra, resulting in improved agreement between experimental data and calculated tuning range. Also shown in the inset to Fig. 3 is the idler beam at a wavelength of $7508 \mathrm{~nm}$, recorded using a pyroelectric camera, confirming high spatial quality with a TEM 00 profile.

We characterized the $\mathrm{OPO}$ with regard to output power by performing power scaling measurements. The results are shown in Fig. 4. For an input pump power $780 \mathrm{~mW}$, we extracted a signal power of $37 \mathrm{~mW}$ at $1155 \mathrm{~nm}$ and an idler power of $12 \mathrm{~mW}$ at $7508 \mathrm{~nm}$, corresponding to a quantum conversion efficiency of $11 \%$. The signal and idler slope efficiencies are estimated to be $8.4 \%$ and $2.3 \%$, respectively. It should also be noted the measured signal and idler powers are not corrected for losses at the input mirror ( 20\%) or residual crystal absorption at the pump 
wavelength. The threshold of the $\mathrm{OPO}$ is measured to be $<300 \mathrm{~mW}$. Using a reflective collimator rather than a $\mathrm{CaF}_{2}$ lens would further improve the practical idler output power.
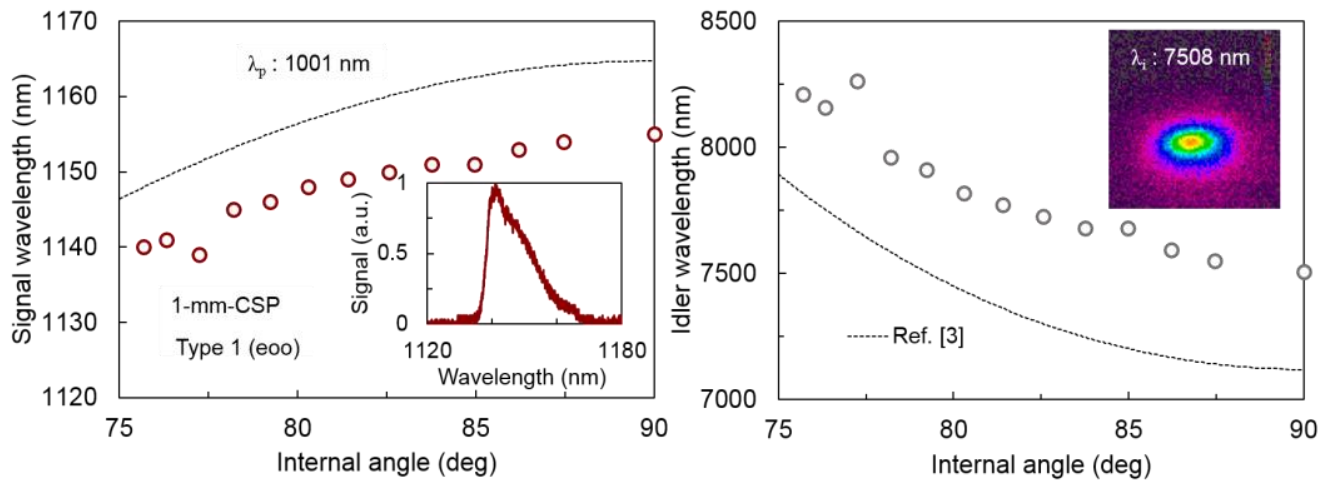

Fig. 3. Angle tuning compared to the Sellmeier predictions from [3]. Left inset: Typical signal spectrum centered at $\sim 1150 \mathrm{~nm}$. Right inset: Idler spatial profile at $7508 \mathrm{~nm}$.

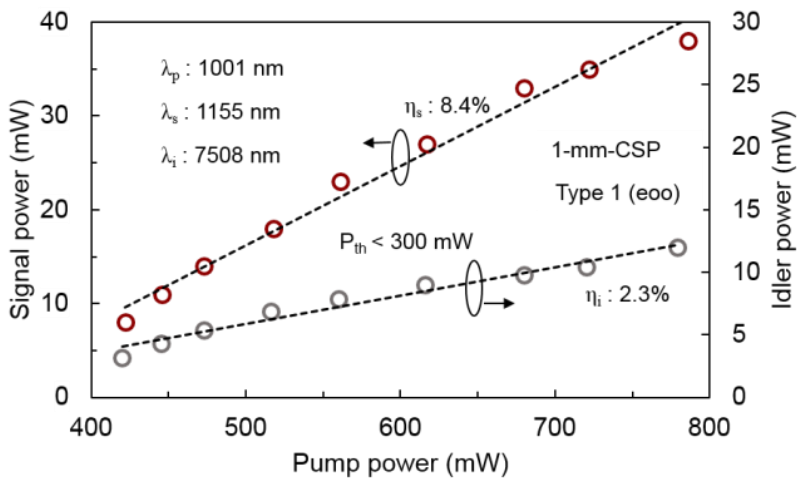

Fig. 4. Signal and idler power scaling of the Ti:sapphire pumped CSP OPO.

In conclusion, we have demonstrated what is to our knowledge the first deep-IR femtosecond OPO based on CSP pumped directly by a KLM Ti:sapphire laser. More than $10 \mathrm{~mW}$ of deep-IR idler power has been generated at 7508 $\mathrm{nm}$, with $>5 \mathrm{~mW}$ available above $8 \mu \mathrm{m}$, the longest wavelengths generated with CSP at such practical powers. Broadband wavelength flexibility across $7508-8210 \mathrm{~nm}$ has been achieved using angle tuning, for the first time, enabling single-device coverage in key parts of the molecular fingerprint region. We have also achieved deep-IR tuning of the OPO using pump tuning range of the KLM Ti:sapphire laser as well as cavity delay tuning, resulting in further extension of spectral coverage in the deep-IR as well as rapid tuning towards both longer and shorter idler wavelengths. The ring cavity design allows a compact physical size with potential for dispersion control using intracavity prisms, which would facilitate transform-limited signal and idler pulses.

\section{References}

[1] G. Edwards, R. Logan, M. Copeland, L. Reinisch, J. Davidson, B. Johnson, R. Maciunas, M. Mendenhall, R. Ossoff, J. Tribble, J. Werkhaven and D. O'day, "Tissue ablation by a free-electron laser tuned to the amide II band," Nature 371, 416 - 419 (1994).

[2] J. Hildenbrand, J. Herbst, J. Wöllenstein, A. Lambrecht, "Explosive detection using infrared laser spectroscopy," Proc. of SPIE 7222, 72220B (2009).

[3] K. T. Zawilski, P. G. Schunemann, T. M. Pollak, D. E. Zelmon, N. C. Fernelius, and F. K. Hopkins, "Growth and characterization of large $\mathrm{CdSiP}_{2}$ single crystals," J. Cryst. Growth 312, 1127-1132 (2010).

[4] S. Chaitanya Kumar, P. G. Schunemann, K. T. Zawilski, and M. Ebrahim-Zadeh, "Advances in ultrafast optical parametric sources for the mid-infrared based on $\mathrm{CdSiP}_{2}$," J. Opt. Soc. Am. B 33, D44-D56 (2016)

[5] Z. Zhang, D. T. Reid, S. Chaitanya Kumar, M. Ebrahim-Zadeh, P. G. Schunemann, K. T. Zawilski, and C. R. Howle, "Femtosecond-laser pumped $\mathrm{CdSiP}_{2}$ optical parametric oscillator producing $100 \mathrm{MHz}$ pulses centered at $6.2 \mu \mathrm{m}$," Opt. Lett. 38, 5110-5113 (2013).

[6] S. Chaitanya Kumar, J. Krauth, A. Steinmann, K. T. Zawilski, P. G. Schunemann, H. Giessen, and M. Ebrahim-Zadeh, "High-power femtosecond mid-infrared optical parametric oscillator at $7 \mu \mathrm{m}$ based on CdSiP 2 ," Opt. Lett. 40, 1398-1401 (2015).

[7] V. Ramaiah-Badarla, S. Chaitanya Kumar, A. Esteban-Martin, K. Devi, K. T. Zawilski, P. G. Schunemann, and M. Ebrahim-Zadeh, "Ti:sapphire-pumped deep-infrared femtosecond optical parametric oscillator based on CdSiP 2 ," Opt. Lett. 41, 1708-1711 (2016).

[8] S. Chaitanya Kumar, A. Esteban-Martin, A. Santana, K. T. Zawilski, P. G. Schunemann, and M. Ebrahim-Zadeh, "Pump-tuned deepinfrared femtosecond optical parametric oscillator across 6-7 $\mu \mathrm{m}$ based on CdSiP 2 ," Opt. Lett. 41, 3355-3358 (2016). 